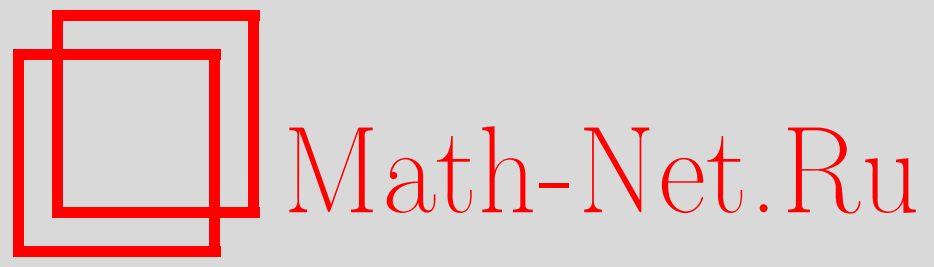

В. П. Маслов, Обобщение метода вторичного квантования на случай специальных тензорных произведений пространств Фока и квантование свободной энергии, Функи. анализ и его прил., 2000, том 34 , выпуск 4, 35-48

DOI: https://doi.org/10.4213/faa324

Использование Общероссийского математического портала MathNet.Ru подразумевает, что вы прочитали и согласны с пользовательским соглашением

http://www. mathnet.ru/rus/agreement

Параметры загрузки:

IP : 34.229 .45 .116

26 апреля 2023 г., 13:05:39

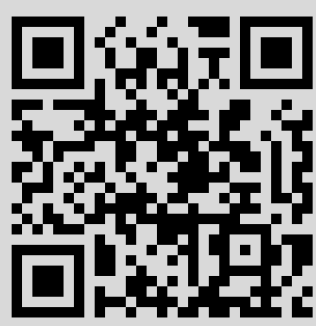


Функциональный анализ и его приложения

2000, т. 34, вып. 4, с. 35-48

УДК 517.9

\title{
Обобщение метода вторичного квантования на случай специальных тензорных произведений пространств Фока и квантование свободной энергии
}

\author{
(C) 2000. В. П. МАСЛОВ
}

В работе проводится осреднение многочастичного оператора Шрёдингера в фоковских пространствах, обобщающее приведенное в статье автора [2]. Показывается, что это есть некоторое новое представление уравнения Шрёдингера, непосредственно обобщающее представление вторичного квантования. Возникает принцип соответствия оператора его символу, который позволяет проквантовать энтропию, а с ней и свободную энергию.

1. Прежде всего мы применим «метод осреднения», рассмотренный в статье [2], для случая бозонов и покажем, что он приводит к обычному вторично квантованному оператору Шрёдингера.

Рассмотрим гильбертово пространство $\mathscr{F}$, элементами которого являются бесконечные последовательности функций

$$
\Psi=\left(\Psi_{0}, \Psi_{1}\left(x_{1}\right), \Psi_{2}\left(x_{1}, x_{2}\right), \ldots\right), \quad \Psi_{N}\left(x_{1}, \ldots, x_{N}\right) \in \mathscr{F}_{N},
$$

где $\mathscr{F}_{N}$ - подпространство пространства $L^{2}(\underbrace{\mathbb{R}^{3} \times \cdots \times \mathbb{R}^{3}}_{N \text { раз }})$, состоящее из элементов, симметричных относительно перестановок аргументов $x_{1}, \ldots, x_{N}$. Скалярное произведение в $\mathscr{F}$ имеет вид

$$
(\Psi, \Phi)=\sum_{N=0}^{\infty} \int d x_{1} \cdots d x_{N} \Psi_{N}^{*}\left(x_{1}, \ldots, x_{N}\right) \Phi_{N}\left(x_{1}, \ldots, x_{N}\right) .
$$

Пространство $\mathscr{F}$ называется симметричным пространством Фока [3]. В пространстве $\mathscr{F}$ определим оператор, называемый осредненным гамильтонианом,

где

$$
\overline{\widehat{\mathscr{H}}}=\sum_{N=0}^{\infty} \widehat{i}_{N} \widehat{H}_{N} \widehat{P}_{N}
$$

$$
\begin{gathered}
\widehat{P}_{N}: \mathscr{F} \rightarrow \mathscr{F}_{N}, \quad \widehat{P}_{N} \Psi=\Psi_{N}, \quad \widehat{i}_{N}: \mathscr{F}_{N} \rightarrow \mathscr{F}, \quad \widehat{i}_{N} \Phi_{N}=(\underbrace{0, \ldots, 0}_{N-1 \text { ра } 3}, \Phi_{N}, 0, \ldots), \\
\widehat{H}_{N}=\sum_{j=1}^{N} T\left(x_{j},-i \hbar \frac{\partial}{\partial x_{j}}\right)+\sum_{1 \leqslant i<j \leqslant N} V\left(x_{i}, x_{j}\right),
\end{gathered}
$$

$T(x,-i \hbar \partial / \partial x)$ - гамильтониан отдельной частицы, а $V(x, y)$ - потенциал взаимодействия между частицами. 
Пусть $\widehat{b}^{ \pm}(x)$ - операторы рождения и уничтожения в $\mathscr{F}$, определяемые стандартным образом [3]:

$$
\begin{aligned}
& \left(\widehat{b}^{+}(x) \Psi\right)_{N}\left(x_{1}, \ldots, x_{N}\right)=\frac{1}{\sqrt{N}} \sum_{j=1}^{N} \delta\left(x-x_{j}\right) \Psi_{N-1}\left(x_{1}, \ldots, x_{j-1}, x_{j+1}, \ldots, x_{N}\right), \\
& \left(\widehat{b}^{-}(x) \Psi\right)_{N-1}\left(x_{1}, \ldots, x_{N}\right)=\sqrt{N} \Psi_{N}\left(x_{1}, \ldots, x_{N-1}, x\right),
\end{aligned}
$$

и $\Psi_{0}=(1,0, \ldots)-$ вакуумный вектор в $\mathscr{F}$. Учитывая формулы

$$
\frac{1}{\sqrt{N !}} \exp \left(-\int d x \widehat{b}^{+}(x) \widehat{b}^{-}(x)\right) \widehat{b}^{-}\left(x_{1}\right) \cdots \widehat{b}^{-}\left(x_{N}\right) \Psi=\Psi_{N}\left(x_{1}, \ldots, x_{N}\right) \Psi_{0},
$$

где число над оператором обозначает порядок его действия [4], и

$$
\widehat{i}_{N} \Phi_{N}\left(x_{1}, \ldots, x_{N}\right)=\frac{1}{\sqrt{N !}} \int d x_{1} \cdots d x_{N} \Phi_{N}\left(x_{1}, \ldots, x_{N}\right) \widehat{b}^{+}\left(x_{1}\right) \cdots \widehat{b}^{+}\left(x_{N}\right) \Psi_{0},
$$

оператор $\overline{\widehat{\mathscr{H}}}$ можно переписать в виде

$$
\begin{aligned}
\overline{\widehat{\mathscr{H}}}=\sum_{N=0}^{\infty} \frac{1}{N !} & \int d x_{1} \cdots d x_{N} \widehat{b}^{+}\left(x_{1}\right) \cdots \widehat{b}^{+}\left(x_{N}\right) \widehat{H}_{N} \\
& \times \exp \left(-\int d \xi \widehat{b}^{+}(\xi) \widehat{b}^{-}(\xi)\right) \widehat{\widehat{b}}^{-}\left(x_{1}\right) \cdots \widehat{b}^{-}\left(x_{N}\right) \equiv \mathscr{H}\left(\widehat{b}^{+}, \widehat{b}^{-}\right) .
\end{aligned}
$$

Функционал $\mathscr{H}\left(b^{*}, b\right)$, в свою очередь, удобно записать, введя в рассмотрение «вспомогательное» пространство Фока $\mathscr{H}_{B}$, элементами которого являются последовательности симметричных относительно перестановок аргументов функций

$$
Y=\left(Y_{0}, Y_{1}\left(x_{1}\right), Y_{2}\left(x_{1}, x_{2}\right), \ldots\right), \quad Y_{N}\left(x_{1}, \ldots, x_{N}\right) \in L^{2}(\underbrace{\mathbb{R}^{3} \times \cdots \times \mathbb{R}^{3}}_{N \text { раз }}) .
$$

(В рассматриваемом случае $\mathscr{H}_{B}$ оказывается совпадающим с $\mathscr{F}$.) Обозначая операторы рождения и уничтожения в $\mathscr{H}_{B}$ через $\widehat{\psi}^{+}(x)$ и $\widehat{\psi}^{-}(x)$, а вакуумный вектор в $\mathscr{H}_{B}$ через $Y_{0}=(1,0, \ldots)$, функционал $\mathscr{H}\left(b^{*}, b\right)$ можно записать в виде

где

$$
\mathscr{H}\left(b^{*}, b\right)=\exp \left(-\int d \xi b^{*}(\xi) b(\xi)\right)\left(Y_{b}, \widehat{H} Y_{b}\right)
$$

$$
\begin{aligned}
Y_{b}= & \exp \left(\int d x \widehat{\psi}^{+}(x) b(x)\right) Y_{0}, \\
\widehat{H}=\int d x \widehat{\psi}^{+}(x) T\left(x,-i \hbar \frac{\partial}{\partial x}\right) \widehat{\psi}^{-}(x) & \\
& +\frac{1}{2} \int d x d y \widehat{\psi}^{+}(x) \widehat{\psi}^{+}(y) V(x, y) \widehat{\psi}^{-}(x) \widehat{\psi}^{-}(y) \equiv H\left(\widehat{\psi}^{+}, \widehat{\psi}^{-}\right) .
\end{aligned}
$$

С помощью формулы коммутации

$$
\widehat{\psi}^{-}(x) \exp \left(\int d \xi \widehat{\psi}^{+}(\xi) b(\xi)\right)=\exp \left(\int d \xi \widehat{\psi}^{+}(\xi) b(\xi)\right)\left(\widehat{\psi}^{-}(x)+b(x)\right)
$$


функционал $\mathscr{H}$ можно привести к виду

$$
\mathscr{H}\left(b^{*}, b\right)=\exp \left(-\int d \xi b^{*}(\xi) b(\xi)\right)\left(Y_{b}, Y_{b}\right) H\left(b^{*}, b\right) .
$$

Вычислив скалярное произведение

$$
\begin{aligned}
\left(Y_{b}, Y_{b}\right) & =\sum_{N=0}^{\infty} \int d x_{1} \cdots d x_{N} \frac{b^{*}\left(x_{1}\right) \cdots b^{*}\left(x_{N}\right)}{\sqrt{N !}} \frac{b\left(x_{1}\right) \cdots b\left(x_{N}\right)}{\sqrt{N !}} \\
& =\exp \left(\int d x b^{*}(x) b(x)\right),
\end{aligned}
$$

получим

$$
\mathscr{H}\left(b^{*}, b\right)=H\left(b^{*}, b\right) .
$$

Таким образом, виковский символ осредненного гамильтониана (1) совпадает с виковским символом вторично квантованного гамильтониана (5).

Если таким способом осреднять единичный оператор, то, как видно из статьи [5], он принимает вид

$$
\begin{aligned}
\overline{\widehat{E}}=\sum_{N=0}^{\infty} \frac{1}{N !} \int d x_{1} \cdots d x_{N} \widehat{b}^{+}\left(x_{1}\right) \cdots \widehat{b}^{+}\left(x_{N}\right) \\
\times \exp \left(-\int d \xi \widehat{b}^{+}(\xi) \widehat{b}^{-}(\xi)\right) \widehat{b}^{-}\left(x_{1}\right) \cdots \widehat{b}^{-}\left(x_{N}\right),
\end{aligned}
$$

а его символ

$$
\begin{aligned}
E\left(b^{*}, b\right)=\sum_{N=0}^{\infty} \frac{1}{N !} \int d x_{1} \cdots d x_{N} b^{*}\left(x_{1}\right) & \cdots b^{*}\left(x_{N}\right) \\
& \times \exp \left(-\int d \xi b^{*}(\xi) b(\xi)\right) b\left(x_{1}\right) \cdots b\left(x_{N}\right)
\end{aligned}
$$

может быть представлен в следующем виде:

$$
E\left(b^{*}, b\right)=\exp \left(-\int d \xi b^{*}(\xi) b(\xi)\right) \operatorname{Sp} \widehat{\rho},
$$

где

$$
\begin{aligned}
\widehat{\rho}=\sum_{N=0}^{\infty} \frac{1}{(N !)^{2}}\left(\int d x b(x) \widehat{\psi}^{+}(x)\right)^{N} & \\
& \quad \times \exp \left(-\int d \xi \widehat{\psi}^{2}+(\xi) \widehat{\psi}^{-}(\xi)\right)\left(\int d y b^{*}(y) \widehat{\psi}^{-}(y)\right)^{N} .
\end{aligned}
$$

Выражение (6) следует из леммы, доказанной в [5], а кроме того, легко может быть получено с помощью результатов [4]. Аналогично, символ осредненного гамильтониана (4) представляется в виде

$$
\mathscr{H}\left(b^{*}, b\right)=\exp \left(-\int d \xi b^{*}(\xi) b(\xi)\right) \operatorname{Sp}(\widehat{\rho} \widehat{H}) .
$$

2. Следуя статье [1], введем осреднение операторов в более общем, чем рассмотренный выше, случае большого числа кластеров и разных типов бозонов. 
Пусть $1 \leqslant k$ - целое число. Пусть $N_{1}, \ldots, N_{k} \geqslant 0$ - целые числа. Рассмотрим гильбертово пространство функций вида

$$
\begin{gathered}
\Psi\left(x_{1}^{1}, j_{1}^{1} ; \ldots ; x_{N_{1}}^{1}, j_{N_{1}}^{1} ; x_{1}^{2}, x_{2}^{2}, j_{1}^{2} ; \ldots ; x_{2 N_{2}-1}^{2}, x_{2 N_{2}}^{2}, j_{N_{2}}^{2} ;\right. \\
\left.\ldots ; x_{1}^{k}, \ldots, x_{k}^{k}, j_{1}^{k} ; \ldots ; x_{k N_{k}-k+1}^{k}, \ldots, x_{k N_{k}}^{k}, j_{N_{k}}^{k}\right),
\end{gathered}
$$

где $x_{p_{l}}^{l} \in \mathbb{T}^{3}, \mathbb{T}^{3}$ - трехмерный тор, длина сторон которого равна $L, j_{q_{l}}^{l}=$ $1, \ldots, \infty$ для всех $l=1, \ldots, k$ и $p_{l}=1, \ldots, l N_{l}, q_{l}=1, \ldots, N_{l}$, причем функции симметричны относительно перестановок любых пар наборов переменных $\left(x_{l p-l+1}^{l}, \ldots, x_{l p}^{l}, j_{p}^{l}\right)$ и $\left(x_{l q-l+1}^{l}, \ldots, x_{l q}^{l}, j_{q}^{l}\right)$ при $l=1, \ldots, k$ и $p, q=1, \ldots, N_{l}$.

Скалярное произведение имеет вид

$$
\begin{gathered}
(\Psi, \Phi) \stackrel{\text { def }}{=} \sum_{j_{1}^{1}=1}^{\infty} \cdots \sum_{j_{N_{1}}^{1}=1}^{\infty} \cdots \sum_{j_{1}^{k}=1}^{\infty} \cdots \sum_{j_{N_{k}}^{k}=1}^{\infty} \int \cdots \int d x_{1}^{1} \cdots d x_{N_{1}}^{1} \cdots d x_{1}^{k} \cdots d x_{k N_{k}}^{k} \\
\times \Psi^{*}\left(x_{1}^{1}, j_{1}^{1} ; \ldots ; x_{N_{1}}^{1}, j_{N_{1}}^{1} ; \ldots ;\right. \\
\left.x_{1}^{k}, \ldots, x_{k}^{k}, j_{1}^{k} ; \ldots ; x_{k N_{k}-k+1}^{k}, \ldots, x_{k N_{k}}^{k}, j_{N_{k}}^{k}\right) \\
\times \Phi\left(x_{1}^{1}, j_{1}^{1} ; \ldots ; x_{N_{1}}^{1}, j_{N_{1}}^{1} ; \ldots ;\right. \\
\left.x_{1}^{k}, \ldots, x_{k}^{k}, j_{1}^{k} ; \ldots ; x_{k N_{k}-k+1}^{k}, \ldots, x_{k N_{k}}^{k}, j_{N_{k}}^{k}\right) .
\end{gathered}
$$

Будем обозначать это пространство $\mathscr{F}_{N_{1}, \ldots, N_{k}}$. Определим бозонное пространство $\mathscr{F}_{N_{1}, \ldots, N_{k}}^{B}$.

ОПРеДЕЛЕНИЕ. Пространство $\mathscr{F}_{N_{1}, \ldots, N_{k}}^{B}$ является подпространством пространства $\mathscr{F}_{N_{1}, \ldots, N_{k}}$ и состоит из тех его элементов, которые симметричны относительно перестановок любых $x_{p}^{l}$ и $x_{q}^{m}$ при $l, m=1, \ldots, k$ и $p=1, \ldots, l N_{l}$, $q=1, \ldots, m N_{m}$. Проектор на это подпространство будет обозначаться через $\widehat{P}_{N_{1}, \ldots, N_{k}}^{B}$,

$$
\widehat{P}_{N_{1}, \ldots, N_{k}}^{B}: \mathscr{F}_{N_{1}, \ldots, N_{k}} \rightarrow \mathscr{F}_{N_{1}, \ldots, N_{k}}^{B} .
$$

Рассмотрим теперь гильбертово пространство $\mathscr{F}$, элементами которого являются бесконечные наборы

$$
\begin{gathered}
\Psi=\left\{\Psi_{N_{1}, \ldots, N_{k}}\right\}, \quad \Psi_{N_{1}, \ldots, N_{k}} \in \mathscr{F}_{N_{1}, \ldots, N_{k}}, N_{1}, \ldots, N_{k}=0,1, \ldots, \\
\|\Psi\|^{2}=\sum_{N_{1}=0}^{\infty} \cdots \sum_{N_{k}=0}^{\infty}\left\|\Psi_{N_{1}, \ldots, N_{k}}\right\|^{2}<\infty .
\end{gathered}
$$

Это пространство является бесконечной прямой суммой:

$$
\mathscr{F}=\bigoplus_{N_{1}, \ldots, N_{k}} \mathscr{F}_{N_{1}, \ldots, N_{k}}
$$

В нем аналогично (3) (см. также [3]) вводятся операторы $\widehat{b}_{l}^{+}\left(x_{1}, \ldots, x_{l}, j\right), \widehat{b}_{l}^{-}\left(x_{1}\right.$, $\left.\ldots, x_{l}, j\right)$ при $l=1, \ldots, k$ - операторы рождения и уничтожения соответственно.

Обозначим оператор проектирования на соответствующее слагаемое прямой суммы через $\widehat{P}_{N_{1}, \ldots, N_{k}}$,

$$
\widehat{P}_{N_{1}, \ldots, N_{k}}: \mathscr{F} \rightarrow \mathscr{F}_{N_{1}, \ldots, N_{k}}, \quad \widehat{P}_{N_{1}, \ldots, N_{k}} \Psi=\Psi_{N_{1}, \ldots, N_{k}},
$$


а оператор вложения $\mathscr{F}_{N_{1}, \ldots, N_{k}}$ в $\mathscr{F}$ через $\widehat{i}_{N_{1}, \ldots, N_{k}}$,

$$
\widehat{i}_{N_{1}, \ldots, N_{k}}: \mathscr{F}_{N_{1}, \ldots, N_{k}} \rightarrow \mathscr{F}, \quad \widehat{i}_{N_{1}, \ldots, N_{k}} \varphi=\left\{\delta_{N_{1} M_{1}} \cdots \delta_{N_{k} M_{k}} \varphi\right\}_{M_{1}, \ldots, M_{k}},
$$

где $\delta_{N M}-$ символ Кронекера.

Введем понятие осредненного оператора. Пусть задан набор операторов $\left\{\widehat{A}_{N}\right\}$ в подпространствах пространства $L^{2}(\underbrace{\mathbb{T}^{3} \times \cdots \times \mathbb{T}^{3}}_{N \text { раз }})$, элементы $\psi\left(x_{1}, \ldots, x_{N}\right)$ которых симметричны относительно перестановок переменных $x_{j}$ и $x_{k}, x_{j} \in \mathbb{T}^{3}$. Поставим в соответствие операторам $\widehat{A}_{N}$ операторы $\widetilde{\widehat{A}}_{N_{1}, \ldots, N_{k}}, N_{1}+2 N_{2}+\cdots+k N_{k}=$ $N$, на подпространствах $\mathscr{F}_{N_{1}, \ldots, N_{k}}^{B}$, действующие как единичные по дискретным переменным $j_{p}^{l}$.

ОПРЕДЕЛЕНИЕ. Осредненным оператором $\overline{\widehat{A}}$ будем называть оператор

$$
\overline{\widehat{A}}=\sum_{N_{1}=0}^{\infty} \cdots \sum_{N_{k}=0}^{\infty}\left(N_{1}+\cdots+k N_{k}\right) ! \widehat{i}_{N_{1}, \ldots, N_{k}} \widetilde{\widehat{A}}_{N_{1}+\cdots+k N_{k}} \widehat{P}_{N_{1}, \ldots, N_{k}}^{B} \widehat{P}_{N_{1}, \ldots, N_{k}}
$$

в пространстве Фока $\mathscr{F}$.

(По поводу регуляризации в случае расходимости и инвариантности относительно выбора коэффициентов суммирования см. [5].)

ЗАмЕчаниЕ. Оператор $\widehat{i}_{N_{1}, \ldots, N_{k}} \widetilde{\widehat{A}}_{N_{1}+\cdots+k N_{k}} \widehat{P}_{N_{1}, \ldots, N_{k}}^{B} \widehat{P}_{N_{1}, \ldots, N_{k}}$ может быть выражен следующим образом:

$$
\begin{aligned}
& \frac{1}{N_{1} ! \cdots N_{k} !} \sum_{j_{1}^{1}=1}^{\infty} \cdots \sum_{j_{N_{1}}^{1}=1}^{\infty} \cdots \sum_{j_{1}^{k}=1}^{\infty} \cdots \sum_{j_{N_{k}}^{k}=1}^{\infty} \int \cdots \int d x_{1}^{1} \cdots d x_{N_{1}}^{1} \cdots d x_{1}^{k} \cdots d x_{k N_{k}}^{k} \\
& \times \widehat{b}_{1}^{2}\left(x_{1}^{1}, j_{1}^{1}\right) \cdots \widehat{b}_{1}^{2}\left(x_{N_{1}}^{1}, j_{N_{1}}^{1}\right) \cdots \widehat{b}_{k}^{2}\left(x_{1}^{k}, \ldots, x_{k}^{k}, j_{k}^{k}\right) \\
& \cdots \widehat{b}_{k}^{+}\left(x_{k N_{k}-k+1}^{k}, \ldots, x_{k N_{k}}^{k}, j_{N_{k}}^{k}\right) \widehat{A}_{N_{1}+\cdots+k N_{k}} \\
& \times \quad \text { Symm } \quad\left\{\widehat{b}_{1}^{-}\left(x_{1}^{1}, j_{1}^{1}\right) \cdots \widehat{b}_{1}^{-}\left(x_{N_{1}}^{1}, j_{N_{1}}^{1}\right)\right. \\
& \left.x_{1}^{1 \cdots x_{N_{1}}^{1} \cdots x_{1}^{k} \cdots x_{k N_{k}}^{k}} \ldots \widehat{b}_{k}^{-}\left(x_{1}^{k}, \ldots, x_{k}^{k}, j_{k}^{k}\right) \cdots \widehat{b}_{k}^{-}\left(x_{k N_{k}-k+1}^{k}, \ldots, x_{k N_{k}}^{k}, j_{N_{k}}^{k}\right)\right\} \\
& \times \exp \left(-\sum_{j=1}^{\infty} \int d z \widehat{b}_{1}^{2}(z, j) \widehat{b}_{1}^{-}(z, j)-\right. \\
& \left.\cdots-\sum_{j=1}^{\infty} \int \cdots \int d z_{1} \cdots d z_{k} \widehat{b}_{k}^{2}\left(z_{1}, \ldots, z_{k}, j\right) \widehat{b}_{k}^{-}\left(z_{1}, \ldots, z_{k}, j\right)\right),
\end{aligned}
$$

где $\operatorname{Symm}_{x_{1} \ldots x_{N}}-$ оператор симметризации по переменным $x_{1}, \ldots, x_{N}$, а числа над операторами обозначают порядок их действия [4]. Выражение (9) в частном случае $k=2$ было получено в [2] и для произвольного $k$ доказывается аналогично.

Исходя из сделанного замечания, символом осредненного оператора $\overline{\widehat{A}}$ будем называть следующий функционал: 


$$
\begin{aligned}
& A\left(b_{1}^{*}\left(x_{1}^{1}, j^{1}\right), b_{1}\left(x_{1}^{1}, j^{1}\right), \ldots, b_{k}^{*}\left(x_{1}^{k}, \ldots, x_{k}^{k}, j^{k}\right), b_{k}\left(x_{1}^{k}, \ldots, x_{k}^{k}, j^{k}\right)\right) \\
& =\sum_{N_{1}=0}^{\infty} \cdots \sum_{N_{k}=0}^{\infty} \frac{\left(N_{1}+2 N_{2}+\cdots+k N_{k}\right) !}{N_{1} ! N_{2} ! \cdots N_{k} !} \\
& \quad \times \sum_{j_{1}^{1}=1}^{\infty} \cdots \sum_{j_{N_{1}}^{1}=1}^{\infty} \cdots \sum_{j_{1}^{k}=1}^{\infty} \cdots \sum_{j_{N_{k}}^{k}=1}^{\infty} \int \cdots \int d x_{1}^{1} \cdots d x_{N_{1}}^{1} \cdots d x_{1}^{k} \cdots d x_{k N_{k}}^{k} \\
& \quad \times b_{1}^{*}\left(x_{1}^{1}, j_{1}^{1}\right) \cdots b_{1}^{*}\left(x_{N_{1}}^{1}, j_{N_{1}}^{1}\right) \cdots b_{k}^{*}\left(x_{1}^{k}, \ldots, x_{k}^{k}, j_{k}^{k}\right) \cdots b_{k}^{*}\left(x_{k N_{k}-k+1}^{k}, \ldots, x_{k N_{k}}^{k}, j_{N_{k}}^{k}\right) \\
& \quad \times \widehat{A}_{N_{1}+\cdots+k N_{k}} \operatorname{Symm}_{x_{1}^{1} \cdots x_{N_{1}}^{1} \cdots x_{1}^{k} \cdots x_{k N_{k}}^{k}}\left\{b_{1}\left(x_{1}^{1}, j_{1}^{1}\right) \cdots b_{1}\left(x_{N_{1}}^{1}, j_{N_{1}}^{1}\right)\right. \\
& \left.\quad \cdots b_{k}\left(x_{1}^{k}, \ldots, x_{k}^{k}, j_{k}^{k}\right) \cdots b_{k}\left(x_{k N_{k}-k+1}^{k}, \ldots, x_{k N_{k}}^{k}, j_{N_{k}}^{k}\right)\right\} \\
& \quad \times \exp \left(-\sum_{j=1}^{\infty} \int d z b_{1}^{*}(z, j) b_{1}(z, j)-\right. \\
& \left.\cdots-\sum_{j=1}^{\infty} \int \cdots \int d z_{1} \cdots d z_{k} b_{k}^{*}\left(z_{1}, \ldots, z_{k}, j\right) b_{k}\left(z_{1}, \ldots, z_{k}, j\right)\right),
\end{aligned}
$$

где $b_{l}\left(\cdot, \ldots, \cdot, j^{l}\right) \in L^{2}\left(\mathbb{T}^{3}\right)$ при $l=1, \ldots, k$ для всех $j^{l}=0,1, \ldots$ и выполнено неравенство

$$
\sum_{j=0}^{\infty} \int \cdots \int d x_{1} \cdots d x_{l} b_{l}^{*}\left(x_{1}, \ldots, x_{l}, j\right) b_{l}\left(x_{1}, \ldots, x_{l}, j\right)<\infty .
$$

Рассмотренному выше набору операторов $\left\{\widehat{A}_{N}\right\}$ отвечает вторично квантованный оператор $\widehat{A}$ в пространстве Фока $\mathscr{H}_{B}$.

\section{Имеет место следующая}

Лемма. Символ осредненного оператора $\overline{\widehat{A}}$ выражается следующим обра3ОМ:

$$
\begin{aligned}
& A\left(b_{1}^{*}\left(x_{1}^{1}, j^{1}\right), b_{1}\left(x_{1}^{1}, j^{1}\right), \ldots, b_{k}^{*}\left(x_{1}^{k}, \ldots, x_{k}^{k}, j^{k}\right), b_{k}\left(x_{1}^{k}, \ldots, x_{k}^{k}, j^{k}\right)\right) \\
& =\operatorname{Sp}(\widehat{A} \widehat{\rho}) \exp \left(-\sum_{j=1}^{\infty} \int d z b_{1}^{*}(z, j) b_{1}(z, j)-\right. \\
& \left.\cdots-\sum_{j=1}^{\infty} \int \cdots \int d z_{1} \cdots d z_{k} b_{k}^{*}\left(z_{1}, \ldots, z_{k}, j\right) b_{k}\left(z_{1}, \ldots, z_{k}, j\right)\right),
\end{aligned}
$$

где $\widehat{\rho}$ является оператором в пространстве $\mathscr{H}_{B}$ и имеет вид

$$
\begin{aligned}
& \widehat{\rho}= \sum_{N_{1}=0}^{\infty} \cdots \sum_{N_{k}=0}^{\infty} \frac{1}{N_{1} ! \cdots N_{k} !} \prod_{l=1}^{k}\left(\sum_{j^{l}=0}^{\infty} \int \cdots \int d x_{1}^{l} d y_{1}^{l} \cdots d x_{l}^{l} d y_{l}^{l}\right. \\
&\left.\times b_{l}\left(x_{1}^{l}, \ldots, x_{l}^{l}, j^{l}\right) \widehat{\psi}^{+}\left(x_{1}^{l}\right) \cdots \widehat{\psi}^{+}\left(x_{l}^{l}\right) b_{l}^{*}\left(y_{1}^{l}, \ldots, y_{l}^{l}, j^{l}\right) \widehat{\hat{\psi}}^{-}\left(y_{1}^{l}\right) \cdots \widehat{\psi}^{-}\left(y_{l}^{l}\right)\right)^{N_{l}} \\
& \times \exp \left(-\int d z \widehat{\widehat{\psi}}^{+}(z){\left.\stackrel{1}{\hat{\psi}^{-}}(z)\right) .}\right.
\end{aligned}
$$


Эта лемма следует из леммы, доказанной в статье [5]. Очевидно, что (10) является обобщением формул (6), (7).

Пусть далее $\overline{\widehat{\mathscr{H}}}$ и $\overline{\widehat{E}}-$ осредненные таким способом гамильтониан (2) и единичный оператор соответственно. Символы этих операторов, согласно лемме, с точностью до множителя равны $\operatorname{Sp} \widehat{\rho}$ и $\operatorname{Sp}(\widehat{H} \widehat{\rho})$. Если оператор $\widehat{\rho}$ считать статистическим оператором (матрицей плотности), то Sp $\widehat{\rho}-$ норма этого оператора, a $\operatorname{Sp}(\widehat{H} \widehat{\rho})$ есть среднее значение гамильтониана, умноженное на $\operatorname{Sp} \widehat{\rho}$. При этом статистическому оператору $\widehat{\rho}$ отвечает энтропия

$$
S=\operatorname{Sp}\left(\widehat{\rho} \ln \left(\frac{\widehat{\rho}}{\operatorname{Sp} \widehat{\rho}}\right)\right)(\operatorname{Sp} \widehat{\rho})^{-1} .
$$

Исходя из аналогии со статистической физикой, будем называть символом осредненной энтропии функционал

$$
\begin{aligned}
& S\left(b_{1}^{*}\left(x_{1}^{1}, j^{1}\right),\right.\left.b_{1}\left(x_{1}^{1}, j^{1}\right), \ldots, b_{k}^{*}\left(x_{1}^{k}, \ldots, x_{k}^{k}, j^{k}\right), b_{k}\left(x_{1}^{k}, \ldots, x_{k}^{k}, j^{k}\right)\right) \\
&=\operatorname{Sp}\left(\widehat{\rho} \ln \left(\frac{\widehat{\rho}}{\operatorname{Sp} \widehat{\rho}}\right)\right) \exp \left(-\sum_{j=1}^{\infty} \int d z b_{1}^{*}(z, j) b_{1}(z, j)-\right. \\
&\left.\quad \ldots-\sum_{j=1}^{\infty} \int \cdots \int d z_{1} \cdots d z_{k} b_{k}^{*}\left(z_{1}, \ldots, z_{k}, j\right) b_{k}\left(z_{1}, \ldots, z_{k}, j\right)\right) .
\end{aligned}
$$

Символу осредненной энтропии поставим в соответствие следующий оператор в пространстве $\mathscr{F}$ :

$$
\overline{\widehat{S}}=\operatorname{reg} S\left(\widehat{b}_{1}^{+}\left(x_{1}^{1}, j^{1}\right), \widehat{b}_{1}^{-}\left(x_{1}^{1}, j^{1}\right), \ldots, \widehat{b}_{k}^{2}\left(x_{1}^{k}, \ldots, x_{k}^{k}, j^{k}\right), \widehat{b}_{k}^{-}\left(x_{1}^{k}, \ldots, x_{k}^{k}, j^{k}\right)\right) .
$$

Для больших $N$ регуляризация проводится с помощью пуантилистической картины [6] (см. конец статьи).

ОПРЕДЕЛЕНИЕ. Числа $\lambda$, такие, что

$$
(\overline{\widehat{\mathscr{H}}}+\theta \overline{\widehat{S}}) \Phi=\lambda \overline{\widehat{E}} \Phi, \quad \Phi \in \mathscr{F}, \Phi \not \equiv 0,
$$

будем называть квантовыми собственными значениями свободной энергии системы бозонов с гамильтонианом $\widehat{H}$ при температуре $\theta$ для $k$ типов кластеров.

3. Теперь аналогично введем осреднение операторов и квантовую свободную энергию для фермионов. Будем считать, что фермионы могут иметь спиновую переменную $s$, которая принимает значения в дискретном конечном множестве $\Sigma$. Рассмотрим гильбертово пространство $\mathscr{F}_{N_{1}, \ldots, N_{k}}$, построенное из функций $(8)$, для которых $x_{p_{l}}^{l} \in \mathbb{T}^{3} \otimes \Sigma$. Далее в этом разделе везде будем использовать обозначение

$$
\int d x=\sum_{s \in \Sigma} \int d \xi,
$$

где $x=(\xi, s), \xi \in \mathbb{T}^{3}, s \in \Sigma$. Определим фермионное пространство $\mathscr{F}_{N_{1}, \ldots, N_{k}}^{F}$.

ОПреДЕЛЕНИЕ. Пространство $\mathscr{F}_{N_{1}, \ldots, N_{k}}^{F}$ является подпространством пространства $\mathscr{F}_{N_{1}, \ldots, N_{k}}$ и состоит из таких элементов $\mathscr{F}_{N_{1}, \ldots, N_{k}}$, которые антисимметричны относительно перестановок любых $x_{p}^{l}$ и $x_{q}^{m}$ при $l, m=1, \ldots, k$ 
и $p=1, \ldots, l N_{l}, q=1, \ldots, m N_{m}$. Проектор на это подпространство будет обозначаться через $\widehat{P}_{N_{1}, \ldots, N_{k}}^{F}$ :

$$
\widehat{P}_{N_{1}, \ldots, N_{k}}^{F}: \mathscr{F}_{N_{1}, \ldots, N_{k}} \rightarrow \mathscr{F}_{N_{1}, \ldots, N_{k}}^{B} .
$$

Введем понятие осредненного оператора в фермионном случае. Пусть в подпространствах пространства $L_{2}(\underbrace{\mathbb{T}^{3} \otimes \Sigma \times \cdots \times \mathbb{T}^{3} \otimes \Sigma}_{N \text { раз }})$ задан набор операторов $\left\{\widehat{A}_{N}\right\}$, элементы $\psi\left(x_{1}, \ldots, x_{N}\right)$ которых антисимметричны относительно перестановок переменных $x_{j}$ и $x_{k}, x_{j} \in \mathbb{T}^{3} \otimes \Sigma$. Поставим в соответствие операторам $\widehat{A}_{N}$ операторы $\overline{\widehat{A}}_{N_{1}, \ldots, N_{k}}, N_{1}+2 N_{2}+\cdots+k N_{k}=N$, на подпространствах $\mathscr{F}_{N_{1}, \ldots, N_{k}}^{F}$, действующие как единичные по дискретным переменным $j_{p}^{l}$.

ОПрЕДЕЛЕНИЕ. Осредненным оператором $\overline{\widehat{A}}$ будем называть оператор

$$
\overline{\widehat{A}}=\sum_{N_{1}=0}^{\infty} \cdots \sum_{N_{k}=0}^{\infty}\left(N_{1}+\cdots+k N_{k}\right) ! \widehat{i}_{N_{1}, \ldots, N_{k}} \overline{\widehat{A}}_{N_{1}+\cdots+k N_{k}} \widehat{P}_{N_{1}, \ldots, N_{k}}^{F} \widehat{P}_{N_{1}, \ldots, N_{k}}
$$

в пространстве Фока $\mathscr{F}$.

ЗАмечАНИЕ. Оператор $\widehat{i}_{N_{1}, \ldots, N_{k}} \widetilde{\widehat{A}}_{N_{1}+\cdots+k N_{k}} \widehat{P}_{N_{1}, \ldots, N_{k}}^{F} \widehat{P}_{N_{1}, \ldots, N_{k}}$ может быть выражен следующим образом:

$$
\begin{aligned}
& \frac{1}{N_{1} ! \cdots N_{k} !} \sum_{j_{1}^{1}=1}^{\infty} \cdots \sum_{j_{N_{1}}^{1}=1}^{\infty} \cdots \sum_{j_{1}^{k}=1}^{\infty} \cdots \sum_{j_{N_{k}}^{k}=1}^{\infty} \int \cdots \int d x_{1}^{1} \cdots d x_{N_{1}}^{1} \cdots d x_{1}^{k} \cdots d x_{k N_{k}}^{k} \\
& \times \widehat{b}_{1}^{2}\left(x_{1}^{1}, j_{1}^{1}\right) \cdots \widehat{b}_{1}^{2}\left(x_{N_{1}}^{1}, j_{N_{1}}^{1}\right) \cdots \widehat{b}_{k}^{2}\left(x_{1}^{k}, \ldots, x_{k}^{k}, j_{k}^{k}\right) \\
& \ldots \widehat{b}_{k}^{2}\left(x_{k N_{k}-k+1}^{k}, \ldots, x_{k N_{k}}^{k}, j_{N_{k}}^{k}\right) \widehat{A}_{N_{1}+\cdots+k N_{k}} \\
& \times \underset{x_{1} x_{1}}{\operatorname{Asymm}}\left\{\widehat{b}_{1}^{-}\left(x_{1}^{1}, j_{1}^{1}\right) \cdots \widehat{b}_{1}^{-}\left(x_{N_{1}}^{1}, j_{N_{1}}^{1}\right)\right. \\
& \begin{array}{r}
x_{1}^{1} \cdots x_{N_{1}}^{1} \cdots x_{1}^{k} \cdots x_{k N_{k}}^{k} \\
\cdots \\
\exp \left(-\sum_{j=1}^{\infty} \int d z \widehat{b}_{k}^{-}\left(x_{1}^{k}, \ldots, x_{k}^{k}(z, j) \widehat{b}_{1}^{-}(z, j)-\right.\right.
\end{array} \\
& \left.\cdots-\sum_{j=1}^{\infty} \int \cdots \int d z_{1} \cdots d z_{k} \widehat{b}_{k}^{2}\left(z_{1}, \ldots, z_{k}, j\right) \widehat{\widehat{b}}_{k}^{-}\left(z_{1}, \ldots, z_{k}, j\right)\right),
\end{aligned}
$$

где Asymm $_{x_{1} \cdots x_{N}}$ - оператор антисимметризации по переменным $x_{1}, \ldots, x_{N}$. Выражение (12) является аналогом выражения (9) в фермионном случае.

Символом осредненного оператора $\widehat{\widehat{A}}$ в фермионном случае будем называть следующий функционал: 


$$
\begin{aligned}
& A\left(b_{1}^{*}\left(x_{1}^{1}, j^{1}\right), b_{1}\left(x_{1}^{1}, j^{1}\right), \ldots, b_{k}^{*}\left(x_{1}^{k}, \ldots, x_{k}^{k}, j^{k}\right), b_{k}\left(x_{1}^{k}, \ldots, x_{k}^{k}, j^{k}\right)\right) \\
& =\sum_{N_{1}=0}^{\infty} \cdots \sum_{N_{k}=0}^{\infty} \frac{\left(N_{1}+2 N_{2}+\cdots+k N_{k}\right) !}{N_{1} ! N_{2} ! \cdots N_{k} !} \\
& \quad \times \sum_{j_{1}^{1}=1}^{\infty} \cdots \sum_{j_{N_{1}}^{1}=1}^{\infty} \cdots \sum_{j_{1}^{k}=1}^{\infty} \cdots \sum_{j_{N_{k}}^{k}=1}^{\infty} \int \cdots \int d x_{1}^{1} \cdots d x_{N_{1}}^{1} \cdots d x_{1}^{k} \cdots d x_{k N_{k}}^{k} \\
& \quad \times b_{1}^{*}\left(x_{1}^{1}, j_{1}^{1}\right) \cdots b_{1}^{*}\left(x_{N_{1}}^{1}, j_{N_{1}}^{1}\right) \cdots b_{k}^{*}\left(x_{1}^{k}, \ldots, x_{k}^{k}, j_{k}^{k}\right) \cdots b_{k}^{*}\left(x_{k N_{k}-k+1}^{k}, \ldots, x_{k N_{k}}^{k}, j_{N_{k}}^{k}\right) \\
& \quad \times \widehat{A}_{N_{1}+\cdots+k N_{k}} \operatorname{Asymm}_{x_{1}^{1} \cdots x_{N_{1}}^{1} \cdots x_{1}^{k} \cdots x_{k N_{k}}^{k}}\left\{b_{1}\left(x_{1}^{1}, j_{1}^{1}\right) \cdots b_{1}\left(x_{N_{1}}^{1}, j_{N_{1}}^{1}\right)\right. \\
& \left.\quad \cdots b_{k}\left(x_{1}^{k}, \ldots, x_{k}^{k}, j_{k}^{k}\right) \cdots b_{k}\left(x_{k N_{k}-k+1}^{k}, \ldots, x_{k N_{k}}^{k}, j_{N_{k}}^{k}\right)\right\} \\
& \quad \times \exp \left(-\sum_{j=1}^{\infty} \int d z b_{1}^{*}(z, j) b_{1}(z, j)-\right. \\
& \left.\cdots-\sum_{j=1}^{\infty} \int \ldots \int d z_{1} \ldots d z_{k} b_{k}^{*}\left(z_{1}, \ldots, z_{k}, j\right) b_{k}\left(z_{1}, \ldots, z_{k}, j\right)\right),
\end{aligned}
$$

где $b_{l}\left(\cdot, \ldots, \cdot, j^{l}\right) \in L^{2}\left(\mathbb{T}^{3 l}\right)$ при $l=1, \ldots, k$ для всех $j^{l}=0,1, \ldots$ и выполнено неравенство

$$
\sum_{j=0}^{\infty} \int \cdots \int d x_{1} \cdots d x_{l} b_{l}^{*}\left(x_{1}, \ldots, x_{l}, j\right) b_{l}\left(x_{1}, \ldots, x_{l}, j\right)<\infty
$$

Рассмотренному выше набору операторов $\left\{\widehat{A}_{N}\right\}$ отвечает вторично квантованный оператор $\widehat{A}$ в фермионном пространстве Фока $\mathscr{H}_{F}$, которое состоит из последовательностей антисимметричных функций и является аналогом пространства $\mathscr{H}_{B}$ (подробно см. в [3]).

В фермионном случае имеет место следующая

ЛЕммА. Символ осредненного оператора $\widehat{\widehat{A}}$ выражается следующим образом:

$$
\begin{gathered}
A\left(b_{1}^{*}\left(x_{1}^{1}, j^{1}\right), b_{1}\left(x_{1}^{1}, j^{1}\right), \ldots, b_{k}^{*}\left(x_{1}^{k}, \ldots, x_{k}^{k}, j^{k}\right), b_{k}\left(x_{1}^{k}, \ldots, x_{k}^{k}, j^{k}\right)\right) \\
=\operatorname{Sp}(\widehat{A} \widehat{\rho}) \exp \left(-\sum_{j=1}^{\infty} \int d z b_{1}^{*}(z, j) b_{1}(z, j)-\right. \\
\left.\cdots-\sum_{j=1}^{\infty} \int \cdots \int d z_{1} \ldots d z_{k} b_{k}^{*}\left(z_{1}, \ldots, z_{k}, j\right) b_{k}\left(z_{1}, \ldots, z_{k}, j\right)\right),
\end{gathered}
$$

где $\widehat{\rho}$ является оператором в пространстве $\mathscr{H}_{F}$ и имеет вид

$$
\begin{aligned}
\widehat{\rho}= & \sum_{N_{1}=0}^{\infty} \cdots \sum_{N_{k}=0}^{\infty} \frac{1}{N_{1} ! \cdots N_{k} !} \sum_{j_{1}^{1}=0}^{\infty} \cdots \sum_{j_{N_{1}}^{1}=0}^{\infty} \cdots \sum_{j_{1}^{k}=0}^{\infty} \cdots \sum_{j_{N_{k}}^{k}=0}^{\infty} \\
& \times \int \cdots \int d x_{1}^{1} d y_{1}^{1} \cdots d x_{N_{1}}^{1} d y_{N_{1}}^{1} \cdots d x_{1}^{k} d y_{1}^{k} \cdots d x_{k N_{k}}^{k} d y_{k N_{k}}^{k} \\
& \times \widehat{\psi}^{+}\left(x_{1}^{1}\right) \cdots \widehat{\psi}^{+}\left(x_{N_{1}}^{1}\right) \cdots \widehat{\psi}^{+}\left(x_{1}^{k}\right) \cdots \widehat{\psi}^{+}\left(x_{k N_{k}}^{k}\right) \widehat{P}_{0}
\end{aligned}
$$




$$
\begin{aligned}
& \times b_{1}\left(x_{1}^{1}, j_{1}^{1}\right) \cdots b_{1}\left(x_{N_{1}}^{1}, j_{N_{1}}^{1}\right) \cdots b_{k}\left(x_{1}^{k}, \ldots, x_{k}^{k}, j_{1}^{k}\right) \cdots b_{k}\left(x_{k N_{k}-k+1}^{k}, \ldots, x_{k N_{k}}^{k}, j_{N_{k}}^{k}\right) \\
& \times b_{1}^{*}\left(y_{1}^{1}, j_{1}^{1}\right) \cdots b_{1}^{*}\left(y_{N_{1}}^{1}, j_{N_{1}}^{1}\right) \cdots b_{k}^{*}\left(y_{1}^{k}, \ldots, y_{k}^{k}, j_{1}^{k}\right) \cdots b_{k}^{*}\left(y_{k N_{k}-k+1}^{k}, \ldots, y_{k N_{k}}^{k}, j_{N_{k}}^{k}\right) \\
& \times \widehat{\psi}^{-}\left(y_{k N_{k}}^{k}\right) \cdots \widehat{\psi}^{-}\left(y_{1}^{k}\right) \cdots \widehat{\psi}^{-}\left(y_{N_{1}}^{1}\right) \cdots \widehat{\psi}^{-}\left(x_{1}^{1}\right),
\end{aligned}
$$

$\widehat{P}_{0}$ - оператор проектирования на вакуумный вектор пространства $\mathscr{H}_{F}$.

Будем называть в фермионном случае символом осредненной энтропии функционал

$$
\begin{aligned}
& S\left(b_{1}^{*}\left(x_{1}^{1}, j^{1}\right), b_{1}\left(x_{1}^{1}, j^{1}\right), \ldots, b_{k}^{*}\left(x_{1}^{k}, \ldots, x_{k}^{k}, j^{k}\right), b_{k}\left(x_{1}^{k}, \ldots, x_{k}^{k}, j^{k}\right)\right) \\
&=\operatorname{Sp}\left(\widehat{\rho} \ln \left(\frac{\widehat{\rho}}{\operatorname{Sp} \widehat{\rho}}\right)\right) \exp \left(-\sum_{j=1}^{\infty} \int d z b_{1}^{*}(z, j) b_{1}(z, j)-\right. \\
&\left.\cdots-\sum_{j=1}^{\infty} \int \cdots \int d z_{1} \cdots d z_{k} b_{k}^{*}\left(z_{1}, \ldots, z_{k}, j\right) b_{k}\left(z_{1}, \ldots, z_{k}, j\right)\right) .
\end{aligned}
$$

Символу осредненной энтропии поставим в соответствие следующий оператор в пространстве $\mathscr{F}$ :

$$
\overline{\widehat{S}}=\operatorname{reg} S\left(\widehat{b}_{1}^{+}\left(x_{1}^{1}, j^{1}\right), \widehat{b}_{1}^{-}\left(x_{1}^{1}, j^{1}\right), \ldots, \widehat{b}_{k}^{+}\left(x_{1}^{k}, \ldots, x_{k}^{k}, j^{k}\right), \widehat{b}_{k}^{-}\left(x_{1}^{k}, \ldots, x_{k}^{k}, j^{k}\right)\right) .
$$

ОПРЕДЕЛЕНИЕ. Числа $\lambda$, такие, что

$$
(\overline{\widehat{\mathscr{H}}}+\theta \overline{\widehat{S}}) \Phi=\lambda \overline{\widehat{E}} \Phi, \quad \Phi \in \mathscr{F}, \Phi \not \equiv 0,
$$

будем называть квантовыми собственными значениями свободной энергии системы фермионов с гамильтонианом $\widehat{H}$ при температуре $\theta$ для $k$ типов кластеров.

4. Пусть в пространстве $\mathscr{F}$ задана система векторов $\left\{\Phi_{n}\right\}$. Умножая уравнения (13) и (11) скалярно на вектор $\Phi_{n}$ и делая подстановку $\Phi=\sum_{m} C_{m} \Phi_{n}$, получим систему уравнений для коэффициентов $C_{m}$

$$
\sum_{m}\left(\Phi_{n},(\overline{\widehat{\mathscr{H}}}+\theta \overline{\widehat{S}}) \Phi_{m}\right) C_{m}=\lambda \sum_{m}\left(\Phi_{n}, \overline{\widehat{E}} \Phi_{m}\right) C_{m}
$$

Естественно, систему векторов $\left\{\Phi_{n}\right\}$ можно выбрать так, чтобы система уравнений (14) упростилась и стала туннельной. Проиллюстрируем этот выбор на примере системы из $N$ фермионов со спином $1 / 2$. Будем считать, что число типов кластеров равно 2. Пусть вторично квантованный гамильтониан фермионов в пространстве $\mathscr{H}_{F}$ имеет вид

$$
\widehat{H}=\sum_{p} \sum_{s=1}^{2} \varepsilon_{p} \widehat{c}_{p s}^{+} \widehat{c}_{p s}^{-}+\sum_{p, q, r, t} \sum_{s, s^{\prime}=1}^{2} V_{p q r t} \widehat{c}_{p s}^{+} \widehat{c}_{q s^{\prime}}^{+} \widehat{c}_{r s^{\prime}}^{+} \widehat{c}_{t s}^{-},
$$

где $s=1,2$ - спиновая переменная, $p, q, r, t$ - трехмерные векторы вида $2 \pi\left(n_{1}, n_{2}, n_{3}\right) / L, n_{1}, n_{2}, n_{3} \in \mathbb{Z}, L-$ длина стороны тора $\mathbb{T}^{3}, \widehat{c}_{p s}^{ \pm}-$операторы рождения и уничтожения фермиона с импульсом $p$ и спином $s$,

$$
\widehat{c}_{p s}^{ \pm}=\frac{1}{L^{3 / 2}} \int d \xi \exp ( \pm i p \xi) \widehat{\psi}^{ \pm}(\xi, s)
$$


$\varepsilon_{p}, V_{p q r t}-$ заданные коэффициенты, причем $V_{p q r t}=0$ при $p+q-r-t \neq 0$, и, кроме того, использовано следующее обозначение:

$$
\sum_{p}=\sum_{n_{1}=-\infty}^{\infty} \sum_{n_{2}=-\infty}^{\infty} \sum_{n_{3}=-\infty}^{\infty}
$$

Поскольку операторы $\overline{\mathscr{H}}, \widehat{\widehat{E}}$ и $\overline{\widehat{S}}$, очевидно, коммутируют с операторами числа частиц данного типа кластеров

$$
\int \cdots \int d x_{1} \cdots d x_{l} \widehat{b}_{l}^{+}\left(x_{1}, \ldots, x_{l}, j\right) \widehat{b}_{l}^{-}\left(x_{1}, \ldots, x_{l}, j\right)
$$

при всех $l=1, \ldots, k$ и $j=0,1, \ldots$, введем в пространстве $\mathscr{F}$ систему векторов вида

$$
\begin{aligned}
\Phi_{\{p \sigma\} M}=\left(\sum_{s} \sum_{s^{\prime}} \iint d \xi d \xi^{\prime} \Phi\left(\xi, s, \xi^{\prime}, s^{\prime}\right) \widehat{b}_{2}^{+}\left(\xi, s, \xi^{\prime}, s^{\prime}, 0\right)\right)^{M} \\
\\
\times \prod_{j=0}^{N-2 M}\left(\int d \zeta u_{p_{j}}(x) \widehat{b}_{1}^{+}\left(\zeta, \sigma_{j}, j\right)\right) \Psi_{0},
\end{aligned}
$$

где $\Psi_{0}-$ вакуумный вектор в пространстве $\mathscr{F}, u_{p}(x)=\exp (i p x) / L^{3 / 2},\{p \sigma\}$ - последовательности пар $\left(p_{j}, \sigma_{j}\right), j=0,1, \ldots$, причем $\left(p_{j}, \sigma_{j}\right) \neq\left(p_{j^{\prime}}, \sigma_{j^{\prime}}\right)$ при $j \neq j^{\prime}, 2 M<N$ и, кроме того,

$$
\begin{gathered}
\Phi\left(\xi, 1, \xi^{\prime}, 2\right)=\sum_{p} \varphi_{p} u_{p}(\xi) u_{-p}\left(\xi^{\prime}\right)=-\Phi\left(\xi^{\prime}, 2, \xi, 1\right) \\
\Phi\left(\xi, 1, \xi^{\prime}, 1\right)=\Phi\left(\xi, 2, \xi^{\prime}, 2\right)=0 .
\end{gathered}
$$

Система векторов (16) в случае, когда $M$ и $N-2 M$ велики, определяет серию, понимаемую в смысле [6]. В пространстве Фока $\mathscr{H}_{F}$ этой серии отвечает следующая система векторов:

$$
\phi_{\{n\}}=\left(\sum_{q} \varphi_{q} \widehat{c}_{q 1}^{+} \widehat{c}_{-q 2}^{+}\right)^{M} \prod_{p}\left(\widehat{c}_{p 1}^{+}\right)^{n_{p 1}}\left(\widehat{c}_{-p 2}^{+}\right)^{n_{-p 2}} Y_{0},
$$

где $\{n\}-$ набор чисел $n_{p s}$, которые могут принимать значения 0 и 1 и при этом $\sum_{p} \sum_{s} n_{p s}=N-2 M$, а $Y_{0}-$ вакуумный вектор пространства $\mathscr{H}_{F}$. Матричные элементы, отвечающие серии (17), легко находятся в термодинамическом пределе, когда $L \rightarrow \infty, N, M \rightarrow \infty$ и при этом $N / L^{3}$ и $M / L^{3}$ стремятся к постоянным значениям. А именно, если при этом наборы $\{n\}$ и $\left\{n^{\prime}\right\}$ так зависят от $L$, что для любой непрерывной функции $f(p)$ суммы

$$
\frac{1}{L^{3}} \sum_{p} f(p) n_{p s}, \quad s=1,2,
$$

имеют предел, равный

$$
\frac{1}{(2 \pi)^{3}} \int d p f(p) n_{s}(p)
$$


то имеет место следующая асимптотика для скалярного произведения:

$$
\begin{aligned}
\left(\phi_{\left\{n^{\prime}\right\}}, \phi_{\{n\}}\right)= & \frac{\left(f^{*} f\right)^{M}(M !)^{2}}{\sqrt{2 \pi S_{2}}} \exp \left(S_{0}\right) \prod_{p} \delta_{n_{p 1}-n_{p 1}^{\prime}+n_{-p 2}-n_{-p 2}^{\prime}} \\
\times & \left(\left(1-\delta_{n_{p 1} 0} \delta_{n_{-p 2} 0} \delta_{n_{-p 2}^{\prime} 1} \delta_{n_{p 1}^{\prime} 1}-\delta_{n_{p 1} 1} \delta_{n_{-p 2} 1} \delta_{n_{-p 2}^{\prime} 0} \delta_{n_{p 1}^{\prime} 0}\right)\right. \\
& \left.\left.\quad+\delta_{n_{p 1} 0} \delta_{n_{-p 2} 0} \delta_{n_{-p 2}^{\prime} 1} \delta_{n_{p 1}^{\prime} 1} \widetilde{\varphi}_{p}+\delta_{n_{p 1} 1} \delta_{n_{-p 2} 1} \delta_{n_{-p 2}^{\prime} 0} \delta_{n_{p 1}^{\prime} 0}\right) \widetilde{\varphi}_{p}^{*}\right)
\end{aligned}
$$

где $\widetilde{\varphi}_{p}=\varphi_{p} / f$, а константа $f$ определяется из уравнения

$$
M=\sum_{p} \frac{\widetilde{\varphi}_{p}^{*} \widetilde{\varphi}_{p}}{1+\widetilde{\varphi}_{p}^{*} \widetilde{\varphi}_{p}} \delta_{n_{p 1} 0} \delta_{n_{-p 2} 0} \delta_{n_{-p 2}^{\prime} 0} \delta_{n_{p 1}^{\prime} 0}-\sum_{p} \delta_{n_{p 1} 0} \delta_{n_{-p 2} 0} \delta_{n_{-p 2}^{\prime} 1} \delta_{n_{p 1}^{\prime} 1}
$$

И

$$
\begin{aligned}
& S_{0}=\sum_{p} \ln \left(1+\widetilde{\varphi}_{p}^{*} \widetilde{\varphi}_{p}\right) \delta_{n_{p 1} 0} \delta_{n_{-p 2} 0} \delta_{n_{-p 2}^{\prime} 0} \delta_{n_{p 1}^{\prime} 0}, \\
& S_{2}=\sum_{p} \frac{\widetilde{\varphi}_{p}^{*} \widetilde{\varphi}_{p}}{\left(1+\widetilde{\varphi}_{p}^{*} \widetilde{\varphi}_{p}\right)^{2}} \delta_{n_{p 1} 0} \delta_{n_{-p 2} 0} \delta_{n_{-p 2}^{\prime} 0} \delta_{n_{p 1}^{\prime} 0} .
\end{aligned}
$$

Если уравнение (19) не имеет решений, то матричный элемент (18) равен нулю.

Отметим теперь, что формальное выражение для оператора $\overline{\widehat{S}}$ и в фермионном, и в бозонном случаях является расходящимся. Это связано с тем, что символ осредненной энтропии не является аналитическим функционалом. Однако в термодинамическом пределе эта расходимость может быть регуляризована. В частности, в рассматриваемом примере при ненулевой температуре после регуляризации система (14) принимает вид

$$
\sum_{\left\{n^{\prime}\right\}} C_{\left\{n^{\prime}\right\}}\left(\phi_{\{n\}}, \widehat{H} \phi_{\left\{n^{\prime}\right\}}\right)=\lambda \sum_{\left\{n^{\prime}\right\}} C_{\left\{n^{\prime}\right\}}\left(\phi_{\{n\}}, \phi_{\left\{n^{\prime}\right\}}\right)+S_{\{n\}} C_{\{n\}},
$$

где

$$
\begin{aligned}
S_{\{n\}}= & \frac{L^{3}}{(2 \pi)^{3}} \int d p\left(\widetilde{n}_{1}(p) \ln \left(\widetilde{n}_{1}(p)\right)+\left(1-\widetilde{n}_{1}(p)\right) \ln \left(1-\widetilde{n}_{1}(p)\right)\right. \\
& \left.\quad+\widetilde{n}_{2}(p) \ln \left(\widetilde{n}_{2}(p)\right)+\left(1-\widetilde{n}_{2}(p)\right) \ln \left(1-\widetilde{n}_{2}(p)\right)\right),
\end{aligned}
$$

а $G_{1}(p), G_{2}(p), R(p)$ являются пределами функций

$$
\begin{gathered}
G_{p 1}=\delta_{n_{p 1} 1}+\delta_{n_{p 1} 0} \delta_{n_{-p 2} 0} \frac{\widetilde{\varphi}_{p}^{*} \widetilde{\varphi}_{p}}{1+\widetilde{\varphi}_{p}^{*} \widetilde{\varphi}_{p}}, \quad G_{p 2}=\delta_{n_{-p 2} 1}+\delta_{n_{p 1} 0} \delta_{n_{-p 2} 0} \frac{\widetilde{\varphi}_{p}^{*} \widetilde{\varphi}_{p}}{1+\widetilde{\varphi}_{p}^{*} \widetilde{\varphi}_{p}}, \\
R_{p}=\delta_{n_{p 1} 0} \delta_{n_{-p 2} 0} \frac{\widetilde{\varphi}_{p}}{1+\widetilde{\varphi}_{p}^{*} \widetilde{\varphi}_{p}},
\end{gathered}
$$

определенных на решетке $(2 \pi / L) \mathbb{Z}^{3}$ при стремлении шага решетки $2 \pi / L$ к 0 .

5. В разд. 4 мы заменили энтропию $\widehat{\widehat{S}}$ на регуляризованный оператор с помощью перехода к пуантилистической картине [6]. Для операторов, отвечающих 
аналитическому символу, такая регуляризация в термодинамическом пределе не изменяет матричных элементов. Покажем это на примере гамильтониана (15).

Пусть $0<\sigma<1$. Введем пуантилистические функции $\widetilde{G}_{p s}$ и $\widetilde{R}_{p}$ следующим образом:

$$
\begin{aligned}
\widetilde{G}_{p s} & =\frac{1}{N^{\sigma}} \sum_{q} G_{q s} \theta\left(\left(6 \pi^{2} N^{\sigma}\right)^{1 / 3} / L-|p-q|\right), \\
\widetilde{R}_{p} & =\frac{1}{N^{\sigma}} \sum_{q} R_{p} \theta\left(\left(6 \pi^{2} N^{\sigma}\right)^{1 / 3} / L-|p-q|\right),
\end{aligned}
$$

где $\theta(\cdot)$ - тета-функция Хевисайда. Рассмотрим средние значения

$$
\frac{\left(\phi_{\{n\}}, \widehat{H} \phi_{\{n\}}\right)}{\left(\phi_{\{n\}}, \phi_{\{n\}}\right)}
$$

гамильтониана (15) на векторах (17). Воспользовавшись формулами (14), (16), (18), получим для (20) следующее выражение:

$$
\begin{gathered}
\left(\sum_{p} \sum_{s=1}^{2} \varepsilon_{p} G_{p s}+\sum_{\substack{p, q \\
p \neq q}} \sum_{s=1}^{2}\left(V_{p q q p}-V_{p q p q}\right) G_{p s} G_{q s}+\sum_{\substack{p, q \\
p \neq-q}}\left(V_{p q q p}+V_{q p p q}\right) G_{p 1} G_{q 2}\right. \\
\left.+\sum_{\substack{p, q \\
p \neq q}}\left(V_{p-p-q q}+V_{-p p q-q}\right) R_{p}^{*} R_{q}\right)\left(1+O\left(\frac{1}{N}\right)\right)
\end{gathered}
$$

Пусть коэффициенты $V_{p q r t}$ в (15) таковы, что

$V_{p q q p}-V_{p q p q}=V_{1}(p, q), \quad V_{p q q p}+V_{q p p q}=V_{2}(p, q), \quad V_{p-p-q q}+V_{-p p q-q}=V_{3}(p, q)$,

где $V_{1}(p, q), V_{2}(p, q), V_{3}(p, q)$ - непрерывные функции. Предположим, что функции на решетке $G_{p s}$ и $R_{p}$ таковы, что в пределе при $L \rightarrow \infty$ для любой непрерывной функции $f(p)$ имеют место соотношения

$$
\begin{aligned}
\lim _{L \rightarrow \infty} \frac{1}{L^{3}} \sum_{p} f(p) G_{p s} & =\frac{1}{(2 \pi)^{3}} \int d p f(p) G_{s}(p), \\
\lim _{L \rightarrow \infty} \frac{1}{L^{3}} \sum_{p} f(p) R_{p} & =\frac{1}{(2 \pi)^{3}} \int d p f(p) R(p) .
\end{aligned}
$$

В этом случае, очевидно, справедливо равенство

$$
\begin{aligned}
& \left(\frac{\left(\phi_{\{n\}}, \widehat{H} \phi_{\{n\}}\right)}{\left(\phi_{\{n\}}, \phi_{\{n\}}\right)}-\left(\sum_{p} \sum_{s=1}^{2} \varepsilon_{p} \widetilde{G}_{p s}+\sum_{\substack{p, q \\
p \neq q}} \sum_{s=1}^{2}\left(V_{p q q p}-V_{p q p q}\right) \widetilde{G}_{p s} \widetilde{G}_{q s}\right.\right. \\
& \left.\left.\quad+\sum_{\substack{p, q \\
p \neq-q}}\left(V_{p q q p}+V_{q p p q}\right) \widetilde{G}_{p 1} \widetilde{G}_{q 2}+\sum_{\substack{p, q \\
p \neq q}}\left(V_{p-p-q q}+V_{-p p q-q}\right) \widetilde{R}_{p}^{*} \widetilde{R}_{q}\right)\right) L^{-3}=O\left(\frac{1}{N}\right) .
\end{aligned}
$$

Таким образом, при замене функций $G_{p s}$ и $R_{p}$ в $(21)$ на пуантилистические функции $\widetilde{G}_{p s}$ и $\widetilde{R}_{p}$ асимптотика матричных элементов гамильтониана в пределе 
не изменяется. Аналогично, в бозонном случае пуантилистическая регуляризация не изменяет матричные элементы операторов с гладким символом.

\section{ЛиТЕРАТУРА}

1. Маслов В. П. Проблема фазовых переходов в сверхтекучей и нормальной жидкостях. ТМФ, 121, № 3, 492-508 (1999).

2. Маслов В. П. Об одном методе осреднения для квантовой задачи многих тел. Функц. анализ и его прил., 33, вып. 4, 50-64 (1999).

3. Березин Ф. А. Метод вторичного квантования. Наука, М., 1986.

4. Маслов В. П. Операторные методы. Наука, М., 1973.

5. Маслов В. П. О способе осреднения для большого числа кластеров. Базовые переходы. ТМФ, 125, № 2 (2000).

6. Maslov V.P. Deterministic quantum chaos for systems of bosons and fermions. Russian J. Math. Phys., 5, No. 4, 473-488 (1998).

Московский государственный институт электроники и математики

Поступило в редакцию 5 октября 2000 г. 